\section{In vivo confocal microscopic evaluation of keratic precipitates and endothelial morphology in Fuchs' uveitis syndrome}

MC Mocan, S Kadayifcilar and M İrkeç

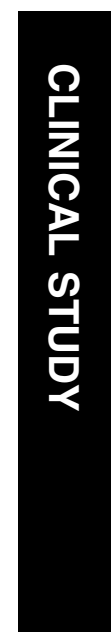

Conclusions FUS is characterized by dendritiform KP and is associated with decreased ECD and altered endothelial cell morphology.

Eye (2012) 26, 119-125; doi:10.1038/eye.2011.268;

published online 4 November 2011

Keywords: Fuchs' uveitis syndrome; keratic precipitates; confocal microscopy

\section{Introduction}

Fuchs' uveitis syndrome (FUS) is an uncommon uveitic entity that was initially described by Georges Weill in 1904 and defined in more detail by Ernst Fuchs in $1906 .{ }^{1-3}$ It is clinically characterized by low-grade intraocular inflammation, keratic precipitates (KP) diffusely distributed over the corneal surface, iris atrophy, and iris hypochromia (or heterochromia in lightly pigmented iridiae). ${ }^{3}$ Associated common findings include cataract, vitritis as evidenced by the presence of vitreous cells and raised intraocular pressure. ${ }^{3}$ Pain is a rare symptom and both posterior synechiae and cystoid macular edema are not observed in the course of FUS. ${ }^{3}$ In the paucity of classical findings, FUS is difficult to diagnose; in fact, only $10-50 \%$ of cases with FUS are identified at initial presentation. ${ }^{4,5}$ Correct diagnosis of FUS is essential as misdiagnosis of this syndrome is associated with the administration of unnecessary and potentially harmful topical and systemic immunosuppressive therapy. ${ }^{5}$

The KP observed in FUS have been noted to have certain morphologic features that may be diagnostically helpful. Under slit-lamp
Department of Ophthalmology, Hacettepe University School of Medicine, Ankara, Turkey

Correspondence: M İrkeç, Department of Üniversitesi, Göz Hastalıkları Anabilim Dalı, Sıhhiye, 06100, Ankara, Turkey Tel: + 903123051777 ; Fax: + 903123094101

E-mail: mirkec@ isnet.net.tr

Received: 9 March 2011 Accepted in revised form: 29 August 2011 Published online: 4 November 2011

The study was presented in part at the 10th International Ocular Inflammation Society Congress in Paris, France (30 May-2 Jun 2009). Ophthalmology, Hacettepe 
biomicroscopy, they are distinguished by their classical, although less commonly observed, fine stellate-shaped appearance or relatively more frequently observed medium-sized precipitates. ${ }^{5}$ Obviously, identification of a distinct KP morphology with FUS would be extremely helpful in correctly identifying eyes with FUS as well as understanding the pathophysiology of this disease.

In vivo confocal microscopy (IVCM) is being used with increasing frequency to characterize the $\mathrm{KP}$ in various uveitic syndromes including FUS. ${ }^{6-11}$ Studies using IVCM have revealed a much more heterogeneity in the morphology of KP than that can be appreciated clinically. ${ }^{12}$ The purpose of this study was to evaluate the endothelial cell layer in detail with respect to the microscopic appearance of KP and to identify whether endothelial cell layer damage was present in patients with FUS using IVCM.

\section{Materials and methods}

\section{Participants}

The study was undertaken at a single university-based hospital setting between January 2006 and January 2011. Informed consent was obtained from all patients and the study was carried out with approval from the Institutional Review Board. All patients included in the study had a diagnosis of FUS. The diagnosis of FUS was established on the detection of the following clinical features by a senior consultant of the Uveitis service (SK), as elaborated in a previous large-scale clinical study: ${ }^{5}$ the presence of low-grade $(\leq+2$ cells in the anterior chamber) anterior chamber reaction not associated with posterior synechiae; nongranulamotous small-medium sized KP as detected with slit-lamp biomicroscopy; diffuse stromal iris atrophy with or without heterochromia; unilateral presentation; absence of pain, photophobia, or ciliary injection; absence of posterior segment involvement including retinal infiltrates, retinal vasculitis, posterior chorioretinal scars or cystoid macular edema, and absence of evidence for another distinct infectious/immunological cause for intraocular inflammation. Patients with a previous history of intraocular trauma or disease were not included. Any patient with a history of herpetic keratitis, signs of corneal involvement in the form of keratitis, granulomatous uveitis, intermediate uveitis, positive chest-X-ray findings, positive serologic evidence of syphilis or any other infectious agent as well as those with positive systemic findings compatible with sarcoidosis or multiple sclerosis were excluded.

The control group consisted of 60 adult patients without any history of eye disease and who had normal ophthalmologic examination findings.

\section{Confocal microscopy in vivo}

IVCM was performed by a single observer (MCM) who was experienced in this technique. Confoscan 3.0 (Nidek Technologies, Vigonza, Italy) attached to an immersion lens (Achroplan $\times 40 / 0.75 \mathrm{~W}$, Zeiss, Göttingen,

Germany) was used to image all corneas. The immersion lens had a working distance of $1.98 \mathrm{~mm}$, a numerical aperture of 0.75 and a front area of $16.61 \mathrm{~mm}^{2}$. The technique is described in detail elsewhere. ${ }^{13}$ IVCM was performed only on the central and paracentral corneal zones of all subjects. As detailed in our previous study, ${ }^{11}$ the corneal endothelium was first examined with slitlamp biomicroscopy to identify the region of the central or the paracentral cornea in which the KP were most densely deposited; IVCM was specifically performed on this region. For each subject, four-to-six corneal scans of the entire corneal thickness including the endothelium were obtained. Particular attention was made to obtain serial sections from the anterior chamber side of the endothelium to the posterior stroma to evaluate the endothelial changes and the KP in full detail. All images of all subjects were obtained in full manual mode of Confoscan 3.0, and automatic light and gain adjustments were not made by the device. Total duration of the confocal microscopic examination lasted for about $2 \mathrm{~min}$ and image acquisition time lasted for $\sim 60 \mathrm{~s}$. The images represented an area of $450 \times 340 \mu \mathrm{m}^{2}$, had a lateral resolution of $1 \mu \mathrm{m}$, and a depth ( $z$ axis) resolution of $10 \mu \mathrm{m}$. The mean magnification obtained was $\times 500$ on a 15-inch display (1024 $\times 768$ pixels) .

\section{Image analysis}

The endothelial cell layer was evaluated in well-focused images of all subjects by a single observer (MI) who was masked to the subjects' eye disease status. Endothelial cell density, cell polymegethism, and cell pleomorphism were evaluated in three images and the results averaged. Endothelial cell counts were performed manually. A boundary box positioned at the image center with fixed dimensions $(200 \mu \mathrm{m} \times 300 \mu \mathrm{m})$ and a size of $0.060 \mathrm{~mm}^{2}$ was used to quantify the endothelial cell densities. The size of the box was kept constant for all study subjects. Cells that overlapped the boundary box were counted at only the superior and the left half of the box. Endothelial cells were selected individually by the operator.

Polymegethism and pleomorphism parameters were calculated automatically using the software program included with Confoscan 3.0 and then critically reviewed by the operator. The KP were further evaluated and classified in four distinct groups: type I (small, round), type II (stippled), type III (dendritiform), and type IV (globular; large, and harboring multiple hyperreflective 
round inclusions in a conglomerate appearance) (Figures 1a-d). When more than one type of KP was observed with IVCM, differentiation between the predominant and the less frequently observed KP was made as 'primary' and 'secondary' KP.

\section{Statistical analysis}

Statistical analyzes were performed using SPSS ver. 15.0 (SPSS Inc., Chicago, IL, USA) software. Student $t$-test was used to compare age and endothelial cell densities of patients with FUS and those of healthy subjects. Of patients with FUS, the endothelial cell densities, and polymegethism and pleomorphism parameters between the affected and normal appearing contralateral eyes were evaluated with the Wilcoxon paired-sample test. A $P$-value of less than 0.05 was considered significant.

\section{Results}

Forty eyes of 40 patients (16 females, 24 males) with a mean age of $32.2 \pm 12.5$ years were included in this study. Nineteen patients had right eye and twenty-one had left eye involvement. Slit-lamp biomicroscopy revealed medium-sized KP in all study subjects (Figure 2a). The mean age of 60 healthy subjects ( $34.8 \pm 14.4$ years) was not significantly different from that of patients with FUS $(P=0.349)$.

In $36(90.0 \%)$ cases with FUS, more than one KP type was observed with IVCM (Figures $2 b$ and c). Type III (dendritiform) KP was the most frequently observed primary KP type $(85.0 \%)$ followed by type II (stippled) $\mathrm{KP}(15.0 \%)$ (Table 1). Among the secondary KP, type II (58.3\%), type IV (globular) (27.8\%), and type III (13.9\%) were more commonly observed. On detailed evaluation of confocal images of all cases, a third KP type could also be observed in nine $(22.5 \%)$ cases; of these, one case had type I (small, round), two cases had type II, and six cases had type IV KP.

Endothelial cell measurements were performed on thirty-six patients with FUS who had not undergone cataract extraction at the time of confocal microscopic evaluation. There was not a significant difference between the mean age of FUS patients who had not undergone cataract surgery and that of normal controls $(P=0.295)$. The mean \pm standard deviation (SD) of endothelial cell density of eyes with FUS (2588 \pm 396 cells $/ \mathrm{mm}^{2}$; range $=1014-3064$ cells $/ \mathrm{mm}^{2}$ ) was significantly lower than that of control subjects $\left(2930 \pm 364\right.$ cells $/ \mathrm{mm}^{2} ;$ range $=1952-3879$ cells $\left./ \mathrm{mm}^{2}\right)$ ( $t$-test; $P<0.001)$. It was noted that a single endothelial cell count of 1014 cells $/ \mathrm{mm}^{2}$ obtained from an eye with FUS was below two standard deviations of the sample mean. Even when this observation was omitted from statistical calculations, the difference between the endothelial cell counts of eyes with FUS $(2606 \pm 297$ cells $/ \mathrm{mm}^{2}$ ) remained significantly lower than that of normal controls $(P<0.001)$. Of the 12 patients with FUS whose contralateral endothelial cell evaluations had been simultaneously performed, there were also statistically

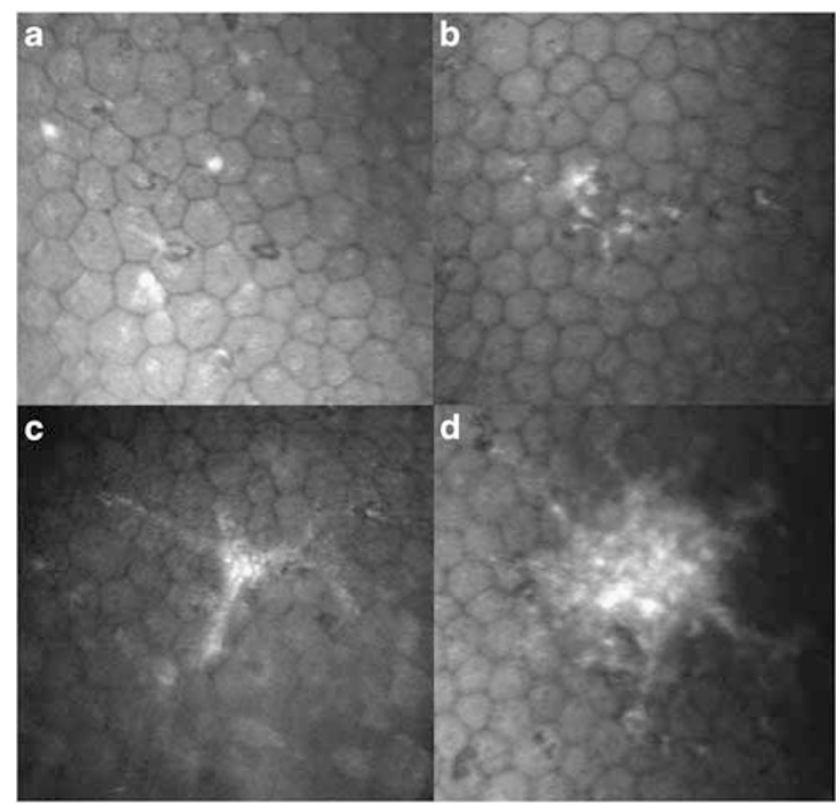

Figure 1 KP morphology as observed by in vivo confocal microscopy. (a) Small, round KP. (b) Stippled KP. (c) Dendritiform KP with a central core and peripheral multiple threadlike extensions. (d) Globular KP; large, round and containing multiple hyperreflective inclusions. 


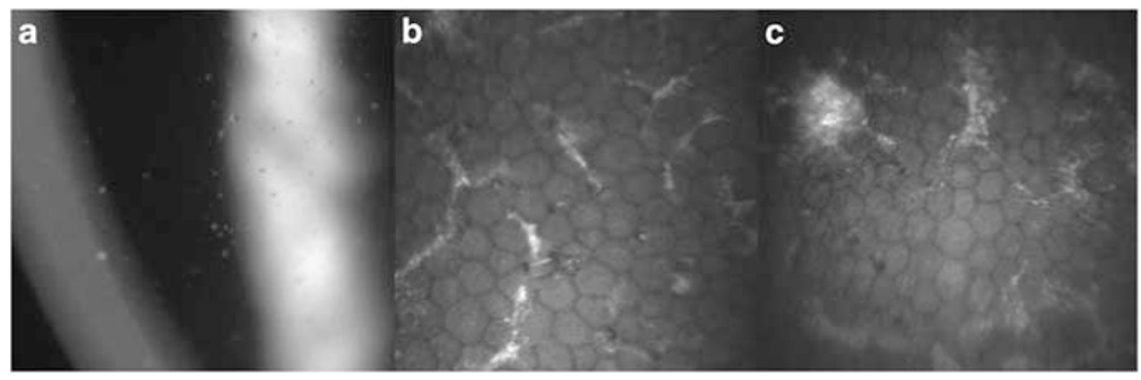

Figure 2 Appearance of KP with slit-lamp examination and IVCM in a patient with FUS (a) Medium and fine KP distributed diffusely on the endothelium as seen with slit-lamp examination. (b) IVCM reveals dendritiform and stippled KP. (c) The occurrence of more than one KP type (globular and dendritiform) in another patient with FUS.

Table 1 The morphology of keratic precipitates observed in patents with Fuchs' uveitis syndrome as imaged with in vivo confocal microscopy

\begin{tabular}{lccccc}
\hline Keratic precipitate predominance & \multicolumn{3}{c}{ Morphology of keratic precipitates } & \multicolumn{3}{c}{ Total } \\
\cline { 2 - 5 } & small round & stipppled & dendritiform & globular & \\
\hline Primary & $0(0.0 \%)$ & $6(15.0 \%)$ & $34(85.0 \%)$ & $0(0.0 \%)$ & 40 \\
Secondary & $0(0.0 \%)$ & $21(58.3 \%)$ & $5(13.9 \%)$ & $10(27.8 \%)$ & 36 \\
\hline
\end{tabular}

Table 2 Comparison of endothelial cell parameters between eyes diagnosed as having Fuchs' uveitis syndrome and clinically normal appearing contralateral eyes of the same subjects

\begin{tabular}{lcccc}
\hline Parameter & Eye with FUS $(\mathrm{n}=12)$ & Contralateral eye $(\mathrm{n}=12)$ & Test & P \\
\hline Endothelial cell density $\left(\right.$ cells $\left./ \mathrm{mm}^{2}\right)$ & $2545 \pm 234$ & $2860 \pm 255$ & Wilcoxon rank-sum test & 0.001 \\
Polymegethism $(\%)$ & $39.0 \pm 6.0$ & $29.2 \pm 3.8$ & Wilcoxon rank-sum test & 0.002 \\
Pleomorphism (\% of hexagonal cells) & $44.1 \pm 6.2$ & $61.2 \pm 9.2$ & Wilcoxon rank-sum test & 0.002 \\
\hline
\end{tabular}

significant differences in the endothelial cell densities, and polymegethism and pleomorphism parameters between the involved eyes with uveitis and the clinically uninvolved contralateral eyes (Table 2). The mean \pm SD of endothelial cell density of the contralateral normal appearing corneas of these 12 patients ( $2860 \pm 255$ cells / $\mathrm{mm}^{2}$; range $=2332-3245$ cells $/ \mathrm{mm}^{2}$ ) was not significantly different than that of healthy subjects' corneas $\left(2930 \pm 364\right.$ cells $/ \mathrm{mm}^{2} ;$ range $=1952-3879$ cells $\left./ \mathrm{mm}^{2}\right)$ ( $t$-test; $P=0.529$ ).

Endothelial blebs were visualized as hyporeflective dark spaces between endothelial cells with IVCM (Figure $3 a$ and $b$ ). The size of the endothelial blebs ranged in size between 3-55 $\mu \mathrm{m}$. Blebs were observed in $37(92.5 \%)$ eyes (small blebs in 21 eyes and large blebs in 16 eyes) overall as well as in $33(91.7 \%)$ corneas of FUS eyes with no prior history of cataract surgery. Of these 33 corneas, $19(57.6 \%)$ harbored smaller $(3-10 \mu \mathrm{m})$ sized blebs (Figure $3 a)$, and $14(42.4 \%)$ had larger $(11-55 \mu \mathrm{m})$ appearing blebs (Figure 3b) that stood out as large dark spots between the unaffected endothelium. The mean endothelium cell density of corneas with small blebs ( $2583 \pm 461$ cells $/ \mathrm{mm}^{2}$ ) was not significantly different from that of corneas that revealed larger dark spots $\left(2626 \pm 353\right.$ cells $\left./ \mathrm{mm}^{2}\right)(P=0.765)$.

$\mathrm{KP}$ or endothelial blebs were not observed in any of the contralateral uninvolved eyes of patients with FUS.

\section{Discussion}

In recent years, the evaluation of KP observed in various uveitic syndromes using IVCM has received considerable attention. ${ }^{7,11,12}$ In vivo confocal microscopic evaluation has highlighted the probable association of certain KP morphologies with distinct uveitic syndromes. The KP observed in FUS has also been studied in prior studies. ${ }^{6,8,9,11}$ Labbé et $a l^{9}$ reported their IVCM findings 


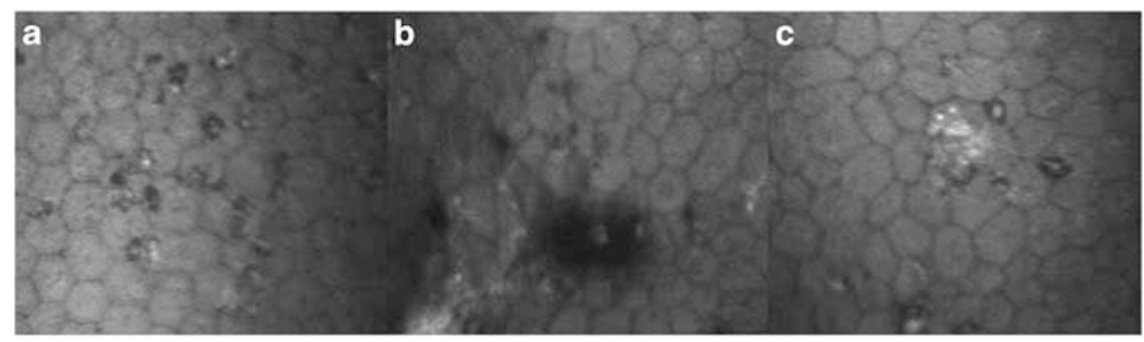

Figure 3 Qualitative alterations in the corneal endothelium of patients with FUS. (a) Small endothelial blebs that present as small $(<10 \mu \mathrm{m})$ hyporeflective spots between the endothelium. (b) Larger $(>10 \mu \mathrm{m})$ blebs that are imaged as dark, hyporeflective spots between the endothelium. (c) Increased polymegethism and pleomorphism associated with endothelial blebs and stippled KP.

on 13 patients with FUS. ${ }^{9}$ The KP observed in that study was reported to be in dendritic shape with a small central body and numerous pseudopodia with accompanying smaller sized dendritic and stippled precipitates. No distinction between primary and secondary KP was made in that study. ${ }^{9}$ Kanavi et $a l^{8}$ evaluated 40 eyes with FUS using IVCM and reported the presence of globular (34 eyes), dendritiform (31 eyes) and stippled (27 eyes) $\mathrm{KP}$. Although a distinction between more frequently KP were not made in that study, the majority $(85 \%)$ of their study subjects revealed $>2 \mathrm{KP}$ types. In the current study, we found a much more higher prevalence of dendritiform KP: in 34 (85\%) eyes, dendritiform KP were the predominant KP observed in FUS corneas and, in further $5(12.5 \%)$ eyes, it was seen as a secondary KP form. Overall dendritiform KP were seen in almost all eyes with FUS, defining it as a very characteristic feature of FUS. The observation of dendritiform KP together with pertinent clinical findings would strongly suggest the diagnosis of FUS, even without the presence of other $\mathrm{KP}$ types such as globular or stippled KP. Conversely, the paucity of dendritiform KP would strongly argue against the diagnosis of FUS as $97.5 \%$ of our cases with FUS presented with dendritiform KP. Dendritiform KP are extremely difficult to appreciate on the basis of clinical grounds, making IVCM an important auxiliary technique in the diagnosis of FUS. Stippled KP were also observed in a total of $27(67.5 \%)$ cases, making it the second most common KP to be associated with FUS. Stippled KP was observed more frequently as a secondary KP in $52.5 \%$ of eyes and accompanying the dendritiform precipitates. The third most common KP type was globular, which was present in $25 \%$ of all eyes and which always presented as a secondary $\mathrm{KP}$ as a single conglomerate inflammatory cellular mass on confocal microscopic images.

In the light of our observations, making the distinction between primary and secondary KP is important from a diagnostic point of view. Dendritiform KP are observed in several uveitic entities including infectious uveitis, ankylosing spondylitis, and Behçet's disease. However, the results of two studies performed on patients with various uveitic syndromes suggest that dendritiform $\mathrm{KP}$ predominate in infectious uveitis and FUS. ${ }^{7,11}$ In infectious uveitis, the coexistence of dendritiform and globular KP is a common finding. ${ }^{10,11}$ On the other hand, in ankylosing spondylitis, dendritiform KP is more often observed as a secondary KP form. Stippled KP, which is observed in eyes with FUS, is also seen in several other entities such as ankylosing spondylitis, Behçet's disease, juvenile idiopathic arthritis, and granulomatous uveitidis. ${ }^{7,11}$ However, it appears to be the predominant $\mathrm{KP}$, only in ankylosing spondylitis. ${ }^{11}$ Therefore, identification of the more prevalent $\mathrm{KP}$ type and evaluation of other coexisting KP in association with the primary KP form may provide more diagnostically valuable information regarding the underlying disorder.

Endothelial cells are permanently damaged by various noxious stimuli including surgical and mechanical trauma, inflammation, and infections, and in association with various dystrophies. ${ }^{14,15}$ Damaged endothelium is characterized by lower cell counts, decreased proportion of hexagonal cells (pleomorphism), and cells with different surface areas (polymegethism). Endothelial cell alterations including decreased cell density has been reported by Pillai et al $^{16}$ in a series of 13 patients diagnosed with unilateral uveitis of various aetiologies (including one case with FUS). In the study by Labbé et $a l,{ }^{9}$ the endothelial cell densities of eyes with FUS and those of the contralateral eyes were not significantly different; however, 8 of the 13 patients with FUS included in their study had already undergone cataract surgery, and paired endothelial cell density comparisons could only be made in 5 patients. In the current study, the comparison of 36 eyes without any history of intraocular surgery with age-matched healthy subjects revealed lower endothelial cell counts in eyes with FUS (Table 2). This difference indicates a limited $(11.7 \%)$ endothelial damage related to FUS. Furthermore, comparison of data 
between 12 eyes with FUS, and their uninvolved contralateral eyes also revealed a significant endothelial cell loss (Table 2). In these 12 eyes, higher percentages of endothelial cell polymegethism and pleomorphism were also observed (Figure 3c). In support of our findings are the results of a prior specular microscopic study by Brooks et al that demonstrated significantly reduced endothelial cell densities associated with altered cellular morphology in patients with FUS. ${ }^{17}$ In a series of eight patients with the diagnosis of FUS, Hasler et $a l^{6}$ demonstrated a lower percentage of hexagonal endothelial cells; in the same study, the decrease in endothelial cell densities of FUS patients was found too small to be significantly different. ${ }^{6}$ The current study has the highest number of patients with FUS $(n=36)$ enrolled for endothelial cell density evaluation. In this regard, the results of our study and those of Brooks et al ${ }^{17}$ suggest that chronic low-grade inflammation and KP have detrimental effects on endothelial cell morphology in FUS with currently unknown implications. When identifying endothelial cell damage related to uveitis, exclusion of coexisting sources of endothelial damage is essential. Perhaps the single most important source of endothelial cell loss in patients with FUS is prior cataract surgery; modern phacoemulsification surgery is associated with an endothelial cell loss of about 7.5-10\%. ${ }^{18,19}$

Endothelial blebs are transient dark spots on the endothelial layer and are considered to be a sign of endothelial stress in response to various forms of endothelial trauma. ${ }^{20}$ They appear between endothelial cells and are considered to represent intercellular edema. ${ }^{20}$ Endothelial blebs in the setting of uveitis were reported in two forms using specular microscopy: small blebs measuring less than the diameter of an endothelium and large blebs ranging in size from 1-4 endothelial cell diameters. ${ }^{16,20}$ In the study by Pillai et al, blebs were more commonly observed in recurrent forms of uveitis. ${ }^{16}$ The findings of previous studies suggest that smaller blebs are localized intra- or inter-cellularly and larger darker blebs are subendothelial in location. ${ }^{16,21}$ The majority $(92.5 \%)$ of corneas evaluated in the current study demonstrated the presence of either small- or large-sized blebs seen as hyporeflective round spots on the endothelial cell layer. The size of the blebs ranged between 3-55 $\mu \mathrm{m}$ and was in agreement with those observed by prior studies. ${ }^{16,21}$ The findings of the current study suggest that bleb type (small vs large) is not associated with a lower endothelial cell count. It should be noted, however, that larger blebs may correspond to the margins of KP and serial sections must be obtained from the anterior chamber side of the endothelium to the stroma to determine the true nature that these large hyporeflective spots.
The patients enrolled in this study represent typical cases with FUS. We included cases with unilateral involvement as FUS is typically unilateral and only $5-10 \%$ of cases are reported to have bilateral involvement. ${ }^{5,22}$

One limitation of IVCM is the difficulty in obtaining images from the peripheral cornea. As one moves the immersion lens closer to the limbus, the lens can only get oblique images of the cornea with insufficient field of view and thus peripheral cornea is inadequately visualized unless the patient makes a very accurate vertical realignment movement so that the peripheral cornea is realigned with the horizontal axis of the immersion lens. For most patients, it is extremely difficult to make this realignment saccade and, in practice, only the central and the paracentral cornea can be imaged with sufficient detail. Typically, the KP observed in the corneas of patients with FUS involve the central and the inferior paracentral cornea and thus can readily be imaged using IVCM. However, in the peripheral cornea with no clinically visible KP, IVCM may demonstrate other types of KP or abnormalities that were not imaged in this study.

Although there were five distinct KP types in the initial article describing KP types in various uveitic syndromes published by the same authors, ${ }^{11}$ the subjects with FUS included in this study did not demonstrate any evidence of the fifth KP type, namely, 'large smooth rounded $\mathrm{KP}^{\prime}$. Therefore, it was decided not to include a $\mathrm{KP}$ type that was not observed in any subjects in this study.

It should be emphasized that IVCM is an auxiliary diagnostic technique and in no means meant to replace the clinical algorithms used in uveitic practice. However, the emerging KP patterns in different uveitic syndromes, including FUS, suggest that characteristic precipitates are suggestive of a number of distinct intraocular inflammatory diseases. ${ }^{7,11}$ The results of this study emphasizes that FUS is almost always associated with dendritiform $\mathrm{KP}$, when the corneas of these patients are imaged with IVCM. If dendritiform KP are not imaged in cases in whom FUS is suspected, the diagnosis of FUS would be highly improbable. The utilization of IVCM would assist in ruling out or supporting the disease in these suspect cases. As such, the utilization of IVCM in the differential diagnosis of uveitic syndromes may aid the clinician in correctly identifying the type of uveitis and thus instituting the appropriate therapy and, in certain instances such as FUS, withholding harmful ones.

In conclusion, the results of our study identify dendritiform type precipitates as being the predominant and characteristic KP in FUS. The endothelium appears to be damaged in patients with FUS in the course of this disease, even in subjects with no prior cataract surgery. 


\section{Summary}

\section{What was known before}

- Fuchs' uveitis syndrome is characterized by the presence of different microscopic keratic precipitates as observed with in vivo confocal microscopy. Fuchs uveitis syndrome was not associated with significant endothelial cell loss.

\section{What this study adds}

- Dendritiform keratic precipitates, as observed with in vivo confocal microscopy, is a highly characteristic feature of Fuchs' uveitis syndrome and is detected in overwhelming majority of cases. Endothelial cell loss, as well as decreased percentage of hexagonal cells, is present in patients with Fuchs' uveitis syndrome.

\section{Conflict of interest}

The authors declare no conflict of interest.

\section{References}

1 Weill G. Über Heterophthalmus. Z Augenheilk 1904; 11(2): 165-176.

2 Fuchs E. Über Komplicationen der Heterochromie. Z Augenheilk 1906; 15(3): 191-212.

3 Mohamed Q, Zamir E. Update on Fuchs' uveitis syndrome. Curr Opin Ophthalmol 2005; 16(6): 356-363.

4 Fearnley IR, Rosenthal AR. Fuchs' heterochromic iridocyclitis revisited. Acta Ophthalmol Scand 1995; 73(2): 166-170.

5 Tugal-Tutkun I, Guney-Tefekli E, Kamaci-Duman F, Corum I. A cross-sectional and longitudinal study of Fuchs uveitis syndrome in Turkish patients. Am J Ophthalmol 2009; 148(4): 510-515 e511.

6 Hasler S, Thiel MA, Becht CN. In vivo confocal microscopy of keratic precipitates in fuchs heterochromic uveitis syndrome. Klin Monbl Augenheilkd 2009; 226(4): 237-240.

7 Kanavi MR, Soheilian M, Naghshgar N. Confocal scan of keratic precipitates in uveitic eyes of various etiologies. Cornea 2010; 29(6): 650-654.

8 Kanavi MR, Soheilian M, Yazdani S, Peyman GA. Confocal scan features of keratic precipitates in Fuchs heterochromic iridocyclitis. Cornea 2010; 29(1): 39-42.

9 Labbe A, Dupas B, Offret H, Baudouin C, Labetoulle M. Evaluation of keratic precipitates and corneal endothelium in Fuchs' heterochromic cyclitis by in vivo confocal microscopy. Br J Ophthalmol 2009; 93(5): 673-677.

10 Mahendradas P, Shetty R, Narayana KM, Shetty BK. In vivo confocal microscopy of keratic precipitates in infectious versus noninfectious uveitis. Ophthalmology 2010; 117(2): 373-380.

11 Mocan MC, Kadayifcilar S, Irkec M. Keratic precipitate morphology in uveitic syndromes including Behcet's disease as evaluated with in vivo confocal microscopy. Eye (Lond) 2009; 23(5): 1221-1227.

12 Wertheim MS, Mathers WD, Planck SJ, Martin TM, Suhler EB, Smith JR et al. In vivo confocal microscopy of keratic precipitates. Arch Ophthalmol 2004; 122(12): 1773-1781.

13 Mocan MC, Durukan I, Irkec M, Orhan M. Morphologic alterations of both the stromal and subbasal nerves in the corneas of patients with diabetes. Cornea 2006; 25(7): 769-773.

14 Tuft SJ, Coster DJ. The corneal endothelium. Eye (Lond) 1990; 4(Pt 3): 389-424.

15 Brooks AM, Grant G, Gillies WE. The use of specular microscopy to investigate unusual findings in the corneal endothelium and its adjacent structures. Aust N Z J Ophthalmol 1988; 16(3): 235-243.

16 Pillai CT, Dua HS, Azuara-Blanco A, Sarhan AR. Evaluation of corneal endothelium and keratic precipitates by specular microscopy in anterior uveitis. Br J Ophthalmol 2000; 84(12): 1367-1371.

17 Brooks AM, Grant G, Robertson IF, Gillies WE. Progressive corneal endothelial cell changes in anterior segment disease. Aust N Z J Ophthalmol 1987; 15(1): 71-78.

18 Green WT, Muir MG. Corneal complications of cataract surgery. Curr Opin Ophthalmol 1994; 5(4): 98-104.

19 Wilczynski M, Drobniewski I, Synder A, Omulecki W. Evaluation of early corneal endothelial cell loss in bimanual microincision cataract surgery (MICS) in comparison with standard phacoemulsification. Eur J Ophthalmol 2006; 16(6): 798-803.

20 Brooks AM, Grant G, Gillies WE. Differential specular microscopy in keratopathy and anterior uveitis. Cornea 1988; 7(2): 105-111.

21 Brooks AM, Grant G, Gillies WE. Differentiation and assessment of corneal endothelial changes associated with diseases of the anterior segment of the eye. Aust N Z J Ophthalmol 1987; 15(1): 65-70.

22 Cunningham Jr ET, Baglivo E. Fuchs heterochromic iridocyclitis - syndrome, disease, or both? Am J Ophthalmol 2009; 148(4): 479-481. 